\title{
On the free boundary value problem for one-dimensional compressible Navier-Stokes equations with constant exterior pressure
}

Ruxu Lian ${ }^{1,2^{*}}$ and Jian $\mathrm{Liu}^{3}$

\section{"Correspondence:}

ruxu.lian.math@gmail.com

${ }^{1}$ College of Mathematics and

Information Science, North China

University of Water Resources and

Electric Power, Zhengzhou, 450011,

P.R. China

${ }^{2}$ Institute of Atmospheric Physics,

Chinese Academy of Sciences,

Beijing, 100029, P.R. China

Full list of author information is

available at the end of the article

\begin{abstract}
In this paper, we consider the free boundary value problem (FBVP) for one-dimensional isentropic compressible Navier-Stokes equations (CNS) with density-dependent viscosity coefficient and constant exterior pressure. Under certain assumptions imposed on the initial data, the global existence and uniqueness of a strong solution to FBVP for CNS are established, in particular, the strong solution tends pointwise to a non-vacuum equilibrium state at an exponential time-rate as the time tends to infinity.
\end{abstract}

Keywords: Navier-Stokes equations; free boundary value problem; density-dependent viscosity coefficient; exterior pressure; strong solution

\section{Introduction}

In the present paper, we consider the free boundary value problem to one-dimensional isentropic compressible Navier-Stokes equations with density-dependent viscosity coefficient for regular initial data in the case that across the free surface the stress tensor is balanced by constant exterior pressure. In general, one-dimensional isentropic compressible Navier-Stokes equations with density-dependent viscosity coefficient can be written as

$$
\left\{\begin{array}{l}
\rho_{t}+(\rho u)_{x}=0, \\
(\rho u)_{t}+\left(\rho u^{2}+P(\rho)\right)_{x}=\left(\mu(\rho) u_{x}\right)_{x}, \quad(x, t) \in R \times[0, T],
\end{array}\right.
$$

where $\rho>0, u$ and $P(\rho)=\rho^{\gamma}(\gamma>1)$ stand for the flow density, velocity and pressure, respectively, and the viscosity coefficient is $\mu(\rho)=\rho^{\alpha}$ with $\alpha>0$. Note here that the case $\gamma=2$ and $\alpha=1$ in (1.1) corresponds to the viscous Saint-Venant system for shallow water.

Recently, there have been much significant progress achieved on the compressible Navier-Stokes equations with density-dependent viscosity coefficients. For instance, the mathematical derivations are obtained in the simulation of flow surface in shallow region $[1,2]$. The existence of solutions for the 2D shallow water equations is investigated by Bresch and Desjardins $[3,4]$. The well-posedness of solutions to the free boundary value problem with initial finite mass and the flow density being connected with the infinite vacuum either continuously or via jump discontinuity is considered by many authors; refer to [5-15] and the references therein. The global existence of classical solutions is shown

@2014 Lian and Liu; licensee Springer. This is an Open Access article distributed under the terms of the Creative Commons Attribution License (http://creativecommons.org/licenses/by/2.0), which permits unrestricted use, distribution, and reproduction in any medium, provided the original work is properly cited. 
by Mellet and Vasseur [16]. The qualitative behaviors of global solutions and dynamical asymptotics of vacuum states are also made, such as the finite time vanishing of finite vacuum or the asymptotical formation of vacuum in large time, the dynamical behaviors of vacuum boundary, the large time convergence to rarefaction wave with vacuum, and the stability of shock profile with large shock strength; refer to [17-22] and the references therein.

In addition, some important progress has been made about free boundary value problems for multi-dimensional compressible viscous Navier-Stokes equations with constant viscosity coefficients for either barotropic or heat-conducive fluids by many authors; for example, in the case that across the free surface stress tensor is balanced by a constant exterior pressure and/or the surface tension, classical solutions with strictly positive densities in the fluid regions to FBVP for CNS (1.1) with constant viscosity coefficients are proved locally in time for either barotropic flows [23-25] or heat-conductive flows [26-28]. In the case that across the free surface the stress tensor is balanced by exterior pressure [25], surface tension [29], or both exterior pressure and surface tension [30], respectively, as the initial data is assumed to be near to a non-vacuum equilibrium state, the global existence of classical solutions with small amplitude and positive densities in fluid region to the FBVP for CNS (1.1) with constant viscosity coefficients is obtained. The global existence of classical solutions to FBVP for compressible viscous and heat-conductive fluids is also established with the stress tensor balanced by the exterior pressure and/or surface tension across the free surface; refer to $[31,32]$ and the references therein.

In this paper, we consider the free boundary value problem (FBVP) for one-dimensional isentropic compressible Navier-Stokes equations with density-dependent viscosity coefficient and constant exterior pressure, and we focus on the existence, regularities and dynamical behaviors of a global strong solution, etc. As $\gamma>1$ we show that the free boundary value problem with regular initial data admits a unique global strong solution which tends pointwise to a non-vacuum equilibrium state at an exponential time-rate as the time tends to infinity (refer to Theorem 2.1 for details).

The rest of the paper is arranged as follows. In Section 2, the main results about the existence and dynamical behaviors of a global strong solution to FBVP with two different initial data for compressible Navier-Stokes equations are stated. Then, some important a priori estimates are given in Section 3 and the theorem is proven in Section 4.

\section{Main results}

We are interested in the global existence and dynamics of the free boundary value problem for (1.1) with the following initial data and boundary conditions:

$$
\left\{\begin{array}{l}
(\rho, u)(x, 0)=\left(\rho_{0}, u_{0}\right), \quad x \in\left[a_{0}, b_{0}\right], \\
\left(\rho^{\gamma}-\rho^{\alpha} u_{x}\right)(a(t), t)=p_{e}, \quad\left(\rho^{\gamma}-\rho^{\alpha} u_{x}\right)(b(t), t)=p_{e}, \quad t>0,
\end{array}\right.
$$

where $x=a(t)$ and $x=b(t)$ are the free boundaries defined by

$$
\begin{cases}\frac{d}{d t} a(t)=u(a(t), t), & a(0)=a_{0}, \\ \frac{d}{d t} b(t)=u(b(t), t), & b(0)=b_{0}, \quad t>0,\end{cases}
$$

and the positive constant $p_{e}$ is the exterior pressure. 
Without loss of generality, the total initial mass is renormalized to be one, i.e.,

$$
\int_{a(t)}^{b(t)} \rho(x, t) d x=\int_{a_{0}}^{b_{0}} \rho_{0}(x) d x:=1
$$

Define

$$
E_{0}:=\frac{1}{2} \int_{a_{0}}^{b_{0}} \rho_{0} u_{0}^{2} d x+\int_{a_{0}}^{b_{0}} \rho_{0}\left(\frac{1}{\gamma-1}\left(\rho_{0}^{\gamma-1}-\bar{\rho}^{\gamma-1}\right)+\bar{\rho}^{\gamma}\left(\rho_{0}^{-1}-\bar{\rho}^{-1}\right)\right) d x
$$

and

$$
\begin{aligned}
E_{1}:= & \frac{1}{2} \int_{a_{0}}^{b_{0}} \rho_{0}\left(u_{0}+\frac{1}{\alpha}\left(\rho_{0}^{\alpha}\right)_{x}\right)^{2} d x \\
& +\int_{a_{0}}^{b_{0}} \rho_{0}\left(\frac{1}{\gamma-1}\left(\rho_{0}^{\gamma-1}-\bar{\rho}^{\gamma-1}\right)+\bar{\rho}^{\gamma}\left(\rho_{0}^{-1}-\bar{\rho}^{-1}\right)\right) d x,
\end{aligned}
$$

where $\bar{\rho}:=p_{e}^{\frac{1}{\gamma}}>0$.

Firstly, we consider the initial data satisfying

$$
\left\{\begin{array}{l}
\inf _{\left[a_{0}, b_{0}\right]} \rho_{0} \geq \rho>0, \quad\left(\rho_{0}, u_{0}\right) \in W^{1, \infty}\left(\left[a_{0}, b_{0}\right]\right), \\
\left(\rho_{0}^{\gamma}-\rho_{0}^{\alpha} u_{0 x}\right)\left(a_{0}\right)=\bar{\rho}^{\gamma}, \quad\left(\rho_{0}^{\gamma}-\rho_{0}^{\alpha} u_{0 x}\right)\left(b_{0}\right)=\bar{\rho}^{\gamma}, \\
\left|\rho_{0}^{\alpha}\left(a_{0}\right)-\bar{\rho}^{\alpha}\right| \leq \delta, \quad\left|\rho_{0}^{\alpha}\left(b_{0}\right)-\bar{\rho}^{\alpha}\right| \leq \delta,
\end{array}\right.
$$

where $\underline{\rho}$ and $\delta>0$ are positive constants, $\delta$ is bounded, and we also consider the other initial data satisfying

$$
\left\{\begin{array}{l}
\inf _{\left[a_{0}, b_{0}\right]} \rho_{0} \geq \rho>0, \quad\left(\rho_{0}, u_{0}\right) \in W^{1, \infty}\left(\left[a_{0}, b_{0}\right]\right), \\
\left(\rho_{0}^{\gamma}-\rho_{0}^{\alpha} u_{0 x}\right)\left(a_{0}\right)=\bar{\rho}^{\gamma}, \quad\left(\rho_{0}^{\gamma}-\rho_{0}^{\alpha} u_{0 x}\right)\left(b_{0}\right)=\bar{\rho}^{\gamma}, \\
\rho_{0}\left(b_{0}\right) \geq \rho_{0}\left(a_{0}\right) \geq \bar{\rho},
\end{array}\right.
$$

note that the compatibility conditions between the initial data and boundary conditions hold. Then we have the global existence and time-asymptotical behavior of a strong solution as follows.

Theorem 2.1 (FBVP) Let $\gamma>1$. Assume that the initial data satisfies (2.6) for $0<\alpha \leq \frac{1}{2}$, and satisfies (2.6) together with

$$
E_{0}^{\frac{1}{2}}\left(E_{0}+2 E_{1}\right)^{\frac{1}{2}} \leq \frac{v}{2(2 \alpha-1)} \bar{\rho}^{\frac{\gamma+2 \alpha-1}{2}}
$$

for $\alpha>\frac{1}{2}$ or the initial data satisfies (2.7) for $0<\alpha \leq \frac{1}{2}$, and satisfies (2.7) together with

$$
E_{0}^{\frac{1}{2}}\left(E_{0}+E_{1}+\left(\rho_{0}^{\gamma}\left(b_{0}\right)-\bar{\rho}^{\gamma}\right) b_{0}-\left(\rho_{0}^{\gamma}\left(a_{0}\right)-\bar{\rho}^{\gamma}\right) a_{0}\right)^{\frac{1}{2}} \leq \frac{v}{2(2 \alpha-1)} \bar{\rho}^{\frac{\gamma+2 \alpha-1}{2}}
$$


for $\frac{1}{2}<\alpha \leq \gamma$, where $v$ is a positive constant. Then there exists a unique global strong solution $(\rho, u, a, b)$ to FBVP (1.1) and (2.1) satisfying, for $T>0$,

$$
\left\{\begin{array}{l}
c \leq \rho \in L^{\infty}\left(0, T ; H^{1}([a(t), b(t)])\right) \cap C^{0}([a(t), b(t)] \times[0, T]), \\
u \in L^{\infty}\left(0, T ; H^{1}([a(t), b(t)])\right) \cap L^{2}\left(0, T ; H^{2}([a(t), b(t)])\right), \\
a(t), b(t) \in H^{1}([0, T]), \quad\left(\rho^{\gamma}-\rho^{\alpha} u_{x}\right) \in L^{\infty}\left(0, T ; L^{2}([a(t), b(t)])\right),
\end{array}\right.
$$

with $c>0$ being a constant independent of time.

If it further holds that $u_{0} \in H^{2}\left(\left[a_{0}, b_{0}\right]\right)$, then $(\rho, u, a, b)$ satisfies

$$
\left\{\begin{array}{l}
(\rho, u) \in C^{0}([a(t), b(t)] \times[0, T]), \\
\rho \in L^{\infty}\left(0, T ; H^{1}([0, a(t)])\right), \quad \rho_{t} \in L^{\infty}\left(0, T ; L^{2}([a(t), b(t)])\right), \\
u \in L^{\infty}\left(0, T ; H^{2}([a(t), b(t)])\right) \cap L^{2}\left(0, T ; H^{3}([a(t), b(t)])\right), \\
u_{t} \in L^{\infty}\left(0, T ; L^{2}([a(t), b(t)])\right) \cap L^{2}\left(0, T ; H^{1}([a(t), b(t)])\right), \\
a(t), b(t) \in H^{2}([0, T]), \quad\left(\rho^{\gamma}-\rho^{\alpha} u_{x}\right) \in C^{0}([0, T] \times([a(t), b(t)])) .
\end{array}\right.
$$

The solution $(\rho, u)$ tends to the non-vacuum equilibrium state exponentially

$$
\|(\rho-\bar{\rho}, u)(\cdot, t)\|_{L^{\infty}([a(t), b(t)])} \leq C_{1} e^{-C_{2} t}, \quad t>0,
$$

where $C_{1}$ and $C_{2}$ are positive constants independent of time.

Remark 2.1 Theorem 2.1 holds for the one-dimensional Saint-Venant model for shallow water, i.e., $\gamma=2, \alpha=1$.

Remark 2.2 The initial constraints (2.8) and (2.9) do not always require that the perturbation of the initial data around the equilibrium state $(\bar{\rho}, 0)$ is small. Indeed, it can be large provided that the state $\bar{\rho}>0$ is large enough.

\section{The a priori estimates}

It is convenient to make use of the Lagrange coordinates in order to establish the $a$ priori estimates. Take the Lagrange coordinates transform

$$
\xi=\int_{a(t)}^{x} \rho(y, t) d y, \quad \tau=t
$$

Since the conservation of total mass holds, the boundaries $x=a(t)$ and $x=b(t)$ are transformed into $\xi=0$ and $\xi=1$, respectively, and the domain $[a(t), b(t)]$ is transformed into $[0,1]$. FBVP (1.1) and (2.1) is reformulated into

$$
\left\{\begin{array}{l}
\rho_{\tau}+\rho^{2} u_{\xi}=0, \\
u_{\tau}+\left(\rho^{\gamma}\right)_{\xi}=\left(\rho^{1+\alpha} u_{\xi}\right)_{\xi}, \\
\left(\rho_{0}, u_{0}\right)=\left(\rho_{0}, u_{0}\right)(\xi), \quad \xi \in[0,1], \\
\left(\rho^{\gamma}-\rho^{1+\alpha} u_{\xi}\right)(0, \tau)=\bar{\rho}^{\gamma}, \quad\left(\rho^{\gamma}-\rho^{1+\alpha} u_{\xi}\right)(1, \tau)=\bar{\rho}^{\gamma}, \quad \tau \in[0, T],
\end{array}\right.
$$

where the initial data satisfies

$$
\left\{\begin{array}{l}
\inf _{[0,1]} \rho_{0} \geq \rho>0, \quad\left(\rho_{0}, u_{0}\right) \in W^{1, \infty}([0,1]), \\
\left(\rho_{0}^{\gamma}-\rho_{0}^{1+\alpha} u_{0 x}\right)(0)=\bar{\rho}^{\gamma}, \quad\left(\rho_{0}^{\gamma}-\rho_{0}^{1+\alpha} u_{0 x}\right)(1)=\bar{\rho}^{\gamma} \\
\left|\rho_{0}^{\alpha}(0)-\bar{\rho}^{\alpha}\right| \leq \delta, \quad\left|\rho_{0}^{\alpha}(1)-\bar{\rho}^{\alpha}\right| \leq \delta
\end{array}\right.
$$


or

$$
\left\{\begin{array}{l}
\inf _{[0,1]} \rho_{0} \geq \underline{\rho}>0, \quad\left(\rho_{0}, u_{0}\right) \in W^{1, \infty}([0,1]), \\
\left(\rho_{0}^{\gamma}-\rho_{0}^{1+\alpha} u_{0 x}\right)(0)=\bar{\rho}^{\gamma}, \quad\left(\rho_{0}^{\gamma}-\rho_{0}^{1+\alpha} u_{0 x}\right)(1)=\bar{\rho}^{\gamma} \\
\rho_{0}(1) \geq \rho_{0}(0) \geq \bar{\rho},
\end{array}\right.
$$

and the consistencies between the initial data and boundary conditions hold.

Next, we deduce the $a$ priori estimates for the solution $(\rho, u)$ to $\operatorname{FBVP}(3.2)$.

Lemma 3.1 Let $T>0$. Under the assumptions of Theorem 2.1, it holds for any strong solution $(\rho, u)$ to FBVP (3.2) that

$$
\begin{gathered}
\int_{0}^{1}\left(\frac{1}{2} u^{2}+\frac{1}{\gamma-1}\left(\rho^{\gamma-1}-\bar{\rho}^{\gamma-1}\right)+\bar{\rho}^{\gamma}\left(\rho^{-1}-\bar{\rho}^{-1}\right)\right) d \xi \\
+\int_{0}^{\tau} \int_{0}^{1} \rho^{1+\alpha} u_{\xi}^{2} d \xi d s=E_{0}, \quad \tau \in[0, T] .
\end{gathered}
$$

Proof Taking the product of $(3.2)_{2}$ with $u$, integrating on $[0,1]$ and using boundary conditions, we have

$$
\begin{aligned}
\frac{1}{2} & \frac{d}{d \tau} \int_{0}^{1} u^{2} d \xi+\int_{0}^{1} \rho^{1+\alpha} u_{\xi}^{2} d \xi \\
& =\int_{0}^{1} u_{\xi}\left(\rho^{\gamma}-\bar{\rho}^{\gamma}\right) d \xi=\int_{0}^{1}\left(\rho^{\gamma-2}-\bar{\rho}^{\gamma} \rho^{-2}\right)\left(-\rho_{\tau}\right) d \xi \\
& =-\frac{d}{d \tau} \int_{0}^{1}\left(\frac{1}{\gamma-1}\left(\rho^{\gamma-1}-\bar{\rho}^{\gamma-1}\right)+\bar{\rho}^{\gamma}\left(\rho^{-1}-\bar{\rho}^{-1}\right)\right) d \xi
\end{aligned}
$$

which leads to (3.5) after the integration with respect to $\tau \in[0, T]$.

Lemma 3.2 Let $T>0$. Under the assumptions of Theorem 2.1, it holds for any strong solution $(\rho, u)$ to FBVP (3.2) with the initial data satisfying (3.3) that

$$
\begin{aligned}
& \int_{0}^{1}\left(\frac{1}{2}\left(u+\frac{1}{\alpha}\left(\rho^{\alpha}\right)_{\xi}\right)^{2}+\frac{1}{\gamma-1}\left(\rho^{\gamma-1}-\bar{\rho}^{\gamma-1}\right)+\bar{\rho}^{\gamma}\left(\rho^{-1}-\bar{\rho}^{-1}\right)\right) d \xi \\
& \quad+\gamma \int_{0}^{\tau} \int_{0}^{1} \rho^{\gamma+2 \alpha-1} \rho_{\xi}^{2} d \xi d s \leq 2 E_{1}, \quad \tau \in[0, T] .
\end{aligned}
$$

As the initial data satisfies (3.4), it holds

$$
\begin{aligned}
\int_{0}^{1}( & \left.\frac{1}{2}\left(u+\frac{1}{\alpha}\left(\rho^{\alpha}\right)_{\xi}\right)^{2}+\frac{1}{\gamma-1}\left(\rho^{\gamma-1}-\bar{\rho}^{\gamma-1}\right)+\bar{\rho}^{\gamma}\left(\rho^{-1}-\bar{\rho}^{-1}\right)\right) d \xi \\
& +\gamma \int_{0}^{\tau} \int_{0}^{1} \rho^{\gamma+2 \alpha-1} \rho_{\xi}^{2} d \xi d s+\left(\rho^{\gamma}(1, \tau)-\bar{\rho}^{\gamma}\right) b(\tau)-\left(\rho^{\gamma}(0, \tau)-\bar{\rho}^{\gamma}\right) a(\tau) \\
& +\frac{\gamma}{\alpha} \int_{0}^{\tau}\left(\rho^{\gamma-\alpha}(1, s)\left(\rho^{\gamma}(1, s)-\bar{\rho}^{\gamma}\right) b(s)-\rho^{\gamma-\alpha}(0, s)\left(\rho^{\gamma}(0, s)-\bar{\rho}^{\gamma}\right) a(s)\right) d s \\
= & E_{1}+\left(\rho_{0}^{\gamma}(1)-\bar{\rho}^{\gamma}\right) b_{0}-\left(\rho_{0}^{\gamma}(0)-\bar{\rho}^{\gamma}\right) a_{0}, \quad \tau \in[0, T],
\end{aligned}
$$

where $a(\tau)$ satisfies $a^{\prime}(\tau)=u(0, \tau)$ and $a(0)=a_{0}, b(\tau)$ satisfies $b^{\prime}(\tau)=u(1, \tau)$ and $b(0)=b_{0}$. 
Proof Multiplying (3.2) $)_{1}$ by $\rho^{\alpha-1}$ leads to

$$
\left(\rho^{\alpha}\right)_{\tau} / \alpha+\rho^{1+\alpha} u_{\xi}=0
$$

which gives

$$
\left(\rho^{\alpha}\right)_{\tau \xi} / \alpha+\left(\rho^{1+\alpha} u_{\xi}\right)_{\xi}=0
$$

Summing (3.2) 1 and (3.10), we deduce

$$
\left(u+\left(\rho^{\alpha}\right)_{\xi} / \alpha\right)_{\tau}+\left(\rho^{\gamma}\right)_{\xi}=0 .
$$

Multiplying (3.11) by $\left(u+\left(\rho^{\alpha}\right)_{\xi} / \alpha\right)$ and integrating the result over $[0,1] \times[0, \tau]$, we have

$$
\begin{aligned}
& \frac{d}{d \tau} \int_{0}^{1}\left(\frac{1}{2}\left(u+\frac{1}{\alpha}\left(\rho^{\alpha}\right)_{\xi}\right)^{2}+\frac{1}{\gamma-1}\left(\rho^{\gamma-1}-\bar{\rho}^{\gamma-1}\right)+\bar{\rho}^{\gamma}\left(\rho^{-1}-\bar{\rho}^{-1}\right)\right) d \xi \\
& \quad+\gamma \int_{0}^{1} \rho^{\gamma+2 \alpha-1} \rho_{\xi}^{2} d \xi+\left(\left(\rho^{\gamma}(1, \tau)-\bar{\rho}^{\gamma}\right) u(1, \tau)-\left(\rho^{\gamma}(0, \tau)-\bar{\rho}^{\gamma}\right) u(0, \tau)\right)=0 .
\end{aligned}
$$

Next, we prove (3.7) in the case that the initial data satisfies (3.3) firstly, to obtain (3.7). We assume a priori that there are constants $\rho_{ \pm}>0$ so that

$$
0<\rho_{-} \leq \rho(\xi, \tau), \bar{\rho} \leq \rho_{+}, \quad(\xi, \tau) \in[0,1] \times[0, T], T>0 .
$$

By means of $(3.2)_{1}$ and the boundary condition, we have

$$
\left(\rho^{\alpha}(1, \tau)-\bar{\rho}^{\alpha}\right)_{\tau}+\left(\rho^{\gamma}(1, \tau)-\bar{\rho}^{\gamma}\right)=0
$$

which yields

$$
\rho^{\alpha}(1, \tau)-\bar{\rho}^{\alpha}=\left(\rho_{0}^{\alpha}(1)-\bar{\rho}^{\alpha}\right) e^{-\int_{0}^{\tau} \frac{\rho^{\gamma}(1, s)-\overline{\bar{\rho}} \gamma}{\rho^{\alpha}(1, s)-\bar{\rho} \alpha} d s} .
$$

From (3.13), we can obtain

$$
\frac{\gamma}{\alpha} \rho_{-}^{\gamma-\alpha} \leq \frac{\rho^{\gamma}(1, \tau)-\bar{\rho}^{\gamma}}{\rho^{\alpha}(1, \tau)-\bar{\rho}^{\alpha}} \leq \frac{\gamma}{\alpha} \rho_{+}^{\gamma-\alpha} .
$$

It holds from (3.5), (3.13), (3.15) and (3.16) that

$$
\begin{aligned}
& \left|\int_{0}^{\tau}\left(\rho^{\gamma}(1, s)-\bar{\rho}^{\gamma}\right) u(1, s) d s\right| \\
& \quad \leq \widehat{C} \int_{0}^{\tau} \rho_{+}^{\gamma-\alpha}\left|\rho^{\alpha}(1, s)-\bar{\rho}^{\alpha}\right|\left(\int_{0}^{1} u^{2} d x+\int_{0}^{1} u_{x}^{2} d x\right)^{\frac{1}{2}} d s \\
& \quad \leq \widehat{C} \int_{0}^{\tau}\left|\rho_{0}^{\alpha}(1)-\bar{\rho}^{\alpha}\right| e^{-\frac{\gamma}{\alpha} \rho_{-}^{\gamma-\alpha}} s\left(\rho_{+}^{2(\gamma-\alpha)}+\int_{0}^{1} u^{2} d \xi+\rho_{-}^{-(1+\alpha)} \int_{0}^{1} \rho^{1+\alpha} u_{x}^{2} d \xi\right) d s \\
& \quad \leq \widehat{C}\left|\rho_{0}^{\alpha}(1)-\bar{\rho}^{\alpha}\right|\left(\rho_{-}^{\alpha-\gamma} \rho_{+}^{2(\gamma-\alpha)}+\rho_{-}^{\alpha-\gamma}+\rho_{-}^{-(1+\alpha)}\right) \leq \frac{1}{2} E_{1},
\end{aligned}
$$


where $\widehat{C}$ is a positive constant independent of time, and we assume that

$$
\left|\rho_{0}^{\alpha}(1)-\bar{\rho}^{\alpha}\right| \leq \min \left\{\frac{\rho_{-}^{\gamma-\alpha} \rho_{+}^{2(\alpha-\gamma)}}{2 \widehat{C}} E_{1}, \frac{\rho_{-}^{\gamma-\alpha}}{2 \widehat{C}} E_{1}, \frac{\rho_{-}^{1+\alpha}}{2 \widehat{C}} E_{1}\right\}:=\delta_{1} .
$$

Using the same method we can obtain

$$
\left|\int_{0}^{\tau}\left(\rho^{\gamma}(0, s)-\bar{\rho}^{\gamma}\right) u(0, s) d s\right| \leq \frac{1}{2} E_{1} .
$$

From (3.12), (3.17) and (3.19), we have (3.7).

Then, we prove (3.8) as the initial data satisfies (3.4), where we have the fact

$$
\begin{aligned}
\int_{0}^{\tau} & \left(\left(\rho^{\gamma}(1, s)-\bar{\rho}^{\gamma}\right) u(1, s)-\left(\rho^{\gamma}(0, s)-\bar{\rho}^{\gamma}\right) u(0, s)\right) d s \\
= & \int_{0}^{\tau}\left(\rho^{\gamma}(1, s)-\bar{\rho}^{\gamma}\right) b^{\prime}(s) d s-\int_{0}^{\tau}\left(\rho^{\gamma}(0, s)-\bar{\rho}^{\gamma}\right) a^{\prime}(s) d s \\
= & \left(\rho^{\gamma}(1, \tau)-\bar{\rho}^{\gamma}\right) b(\tau)-\left(\rho_{0}^{\gamma}(1)-\bar{\rho}^{\gamma}\right) b_{0}-\left(\rho^{\gamma}(0, \tau)-\bar{\rho}^{\gamma}\right) a(\tau)+\left(\rho_{0}^{\gamma}(0)-\bar{\rho}^{\gamma}\right) a_{0} \\
& \quad-\frac{\gamma}{\alpha} \int_{0}^{\tau} b(s) \rho^{\gamma-\alpha}(1, s)\left(\rho^{\alpha}(1, s)\right)_{\tau} d s+\frac{\gamma}{\alpha} \int_{0}^{\tau} a(s) \rho^{\gamma-\alpha}(0, s)\left(\rho^{\alpha}(0, s)\right)_{\tau} d s \\
= & \left(\rho^{\gamma}(1, \tau)-\bar{\rho}^{\gamma}\right) b(\tau)-\left(\rho^{\gamma}(0, \tau)-\bar{\rho}^{\gamma}\right) a(\tau)-\left(\rho_{0}^{\gamma}(1)-\bar{\rho}^{\gamma}\right) b_{0}+\left(\rho_{0}^{\gamma}(0)-\bar{\rho}^{\gamma}\right) a_{0} \\
& +\frac{\gamma}{\alpha} \int_{0}^{\tau} \rho^{\gamma-\alpha}(1, s)\left(\rho^{\gamma}(1, s)-\bar{\rho}^{\gamma}\right) b(s) d s \\
& \quad-\frac{\gamma}{\alpha} \int_{0}^{\tau} \rho^{\gamma-\alpha}(0, s)\left(\rho^{\gamma}(0, s)-\bar{\rho}^{\gamma}\right) a(s) d s .
\end{aligned}
$$

Applying equation (3.2) $)_{1}$ and the boundary condition, we have that

$$
\left(\rho^{\alpha}(1, \tau)-\rho^{\alpha}(0, \tau)\right)_{\tau}+\left(\rho^{\gamma}(1, \tau)-\rho^{\gamma}(0, \tau)\right)=0
$$

and

$$
\rho^{\alpha}(1, \tau)-\rho^{\alpha}(0, \tau)=\left(\rho_{0}^{\alpha}(1)-\rho_{0}^{\alpha}(0)\right) e^{-\int_{0}^{\tau} \frac{\rho^{\gamma}(1, s)-\rho^{\gamma}(0, s)}{\rho^{\alpha}(1, s)-\rho^{\alpha}(0, s)} d s} \geq 0,
$$

which together with

$$
\begin{aligned}
& \rho^{\alpha}(1, \tau)-\bar{\rho}^{\alpha}=\left(\rho_{0}^{\alpha}(1)-\bar{\rho}^{\alpha}\right) e^{-\int_{0}^{\tau} \frac{\rho^{\gamma}(1, s)-\bar{\rho} \gamma}{\rho^{\alpha}(1, s)-\bar{\rho}^{\alpha}} d s} \geq 0, \\
& \rho^{\alpha}(0, \tau)-\bar{\rho}^{\alpha}=\left(\rho_{0}^{\alpha}(0)-\bar{\rho}^{\alpha}\right) e^{-\int_{0}^{\tau} \frac{\rho^{\gamma}(0, s)-\bar{\rho}^{\gamma} \gamma}{\rho^{\alpha}(0, s)-\bar{\rho}^{\alpha}} d s} \geq 0
\end{aligned}
$$

and

$$
b(\tau)-a(\tau)=\int_{0}^{1} \frac{1}{\rho(\zeta, \tau)} d \zeta>0
$$

gives rise to (3.8). 
Lemma 3.3 Let $T>0$. Under the assumptions of Theorem 2.1, it holds

$$
0<\rho_{*} \leq \rho(\xi, \tau) \leq \rho^{*}, \quad(\xi, \tau) \in[0,1] \times[0, T], T>0,
$$

where $\rho_{*}$ and $\rho^{*}$ are positive constants independent of time.

Proof Define

$$
\varphi(\rho):=\frac{1}{\gamma-1}\left(\rho^{\gamma-1}-\bar{\rho}^{\gamma-1}\right)+\bar{\rho}^{\gamma}\left(\rho^{-1}-\bar{\rho}^{-1}\right)
$$

and

$$
\psi(\rho):=\int_{\bar{\rho}}^{\rho} \varphi(\eta)^{\frac{1}{2}} \eta^{\alpha-1} d \eta .
$$

It is easy to verify that $\varphi(\rho) \geq 0$ and $\psi^{\prime}(\rho) \geq 0$. In addition, it holds as $\rho \rightarrow+\infty$ that

$$
\begin{aligned}
\lim _{\rho \rightarrow+\infty} \psi(\rho) & \rightarrow(\gamma-1)^{-\frac{1}{2}} \lim _{\rho \rightarrow+\infty} \int_{\bar{\rho}}^{\rho} \eta^{\frac{\gamma+2 \alpha-3}{2}} d \eta \\
& =\lim _{\rho \rightarrow+\infty} \frac{2}{(\gamma+2 \alpha-1) \sqrt{\gamma-1}}\left(\rho^{\frac{\gamma+2 \alpha-1}{2}}-\bar{\rho}^{\frac{\gamma+2 \alpha-1}{2}}\right) \rightarrow+\infty,
\end{aligned}
$$

and as $\rho \rightarrow 0$ that

$$
\begin{aligned}
\lim _{\rho \rightarrow 0} \psi(\rho) & \rightarrow \lim _{\rho \rightarrow 0} \int_{\bar{\rho}}^{\rho} \bar{\rho}^{\frac{\gamma}{2}} \eta^{\alpha-\frac{3}{2}} d \eta \\
& = \begin{cases}\lim _{\rho \rightarrow 0} \frac{2}{2 \alpha-1} \bar{\rho}^{\frac{\gamma}{2}}\left(\rho^{\alpha-\frac{1}{2}}-\bar{\rho}^{\alpha-\frac{1}{2}}\right), & \alpha \neq \frac{1}{2}, \\
\lim _{\rho \rightarrow 0} \bar{\rho}^{\frac{\gamma}{2}}(\ln \rho-\ln \bar{\rho}), & \alpha=\frac{1}{2}\end{cases} \\
& \rightarrow \begin{cases}-\infty, & 0<\alpha \leq \frac{1}{2}, \\
-\frac{2}{2 \alpha-1} \bar{\rho}^{\frac{\gamma+2 \alpha-1}{2}}, & \alpha>\frac{1}{2} .\end{cases}
\end{aligned}
$$

As the initial data satisfies (3.3), it follows from (3.5) and (3.7) that

$$
\begin{aligned}
|\psi(\rho(\xi, \tau))| & \leq|\psi(\rho(1, \tau))|+\left|\int_{0}^{1} \partial_{\xi} \psi(\rho) d \xi\right| \\
& \leq \widetilde{C}\left|\rho^{\alpha}(1, \tau)-\bar{\rho}^{\alpha}\right|+\left|\int_{0}^{1} \varphi(\rho)^{\frac{1}{2}} \rho^{\alpha-1} \rho_{\xi} d \xi\right| \\
& \leq \widetilde{C}\left|\rho_{0}^{\alpha}(1)-\bar{\rho}^{\alpha}\right|+\frac{1}{v}\left(\int_{0}^{1} \varphi(\rho) d \xi\right)^{\frac{1}{2}}\left(\int_{0}^{1} \frac{1}{\alpha^{2}}\left(\rho^{\alpha}\right)_{\xi}^{2} d \xi\right)^{\frac{1}{2}} \\
& \leq \frac{4}{v} E_{0}^{\frac{1}{2}}\left(E_{0}+2 E_{1}\right)^{\frac{1}{2}}
\end{aligned}
$$

where $\widetilde{C}$ and $v$ are positive constants independent of time, and we assume that

$$
\left|\rho_{0}^{\alpha}(1)-\bar{\rho}^{\alpha}\right| \leq \min \left\{\frac{2}{\widetilde{C} \nu} E_{0}^{\frac{1}{2}}\left(E_{0}+2 E_{1}\right)^{\frac{1}{2}}, \delta_{1}\right\}:=\delta .
$$


As $0<\alpha \leq \frac{1}{2}$ and as $\alpha>\frac{1}{2}$, from the condition

$$
E_{0}^{\frac{1}{2}}\left(E_{0}+2 E_{1}\right)^{\frac{1}{2}} \leq \frac{v}{2(2 \alpha-1)} \bar{\rho}^{\frac{\gamma+2 \alpha-1}{2}}
$$

we can find that there are two positive constants $\rho_{*}$ and $\rho^{*}$ independent of time and choose

$$
\rho_{-}=\frac{\rho_{*}}{2}, \quad \rho_{+}=2 \rho^{*},
$$

such that

$$
0<\rho_{-}<\rho_{*} \leq \rho(\xi, \tau) \leq \rho^{*}<\rho_{+}, \quad(\xi, \tau) \in[0,1] \times[0, T], T>0 .
$$

As the initial data satisfies (3.4), it follows from (3.5) and (3.8) that

$$
\begin{aligned}
|\psi(\rho(\xi, \tau))| \leq & \left|\int_{0}^{1} \psi(\rho) d \xi\right|+\left|\int_{0}^{1} \partial_{\xi} \psi(\rho) d \xi\right| \\
\leq & \left|\int_{0}^{1} \varphi(\rho)^{\frac{1}{2}} \rho^{\alpha} d \xi\right|+\left|\int_{0}^{1} \varphi(\rho)^{\frac{1}{2}} \rho^{\alpha-1} \rho_{\xi} d \xi\right| \\
\leq & \frac{1}{v}\left(\int_{0}^{1} \varphi(\rho) d \xi\right)^{\frac{1}{2}}\left(\int_{0}^{1} \rho^{2 \alpha} d \xi\right)^{\frac{1}{2}} \\
& +\frac{1}{v}\left(\int_{0}^{1} \varphi(\rho) d \xi\right)^{\frac{1}{2}}\left(\int_{0}^{1} \frac{1}{\alpha^{2}}\left(\rho^{\alpha}\right)_{\xi}^{2} d \xi\right)^{\frac{1}{2}} \\
\leq & \frac{2}{v}\left(\int_{0}^{1} \varphi(\rho) d \xi\right)^{\frac{1}{2}}\left(\int_{0}^{1} \frac{1}{\alpha^{2}}\left(\rho^{\alpha}\right)_{\xi}^{2} d \xi\right)^{\frac{1}{2}} \\
\leq & \frac{4}{v} E_{0}^{\frac{1}{2}}\left(E_{0}+E_{1}+\left(\rho_{0}^{\gamma}\left(b_{0}\right)-\bar{\rho}^{\gamma}\right) b_{0}-\left(\rho_{0}^{\gamma}\left(a_{0}\right)-\bar{\rho}^{\gamma}\right) a_{0}\right)^{\frac{1}{2}}
\end{aligned}
$$

where $v$ is a positive constant independent of time, and we use the fact that

$$
\left\|\rho^{\alpha}(\xi, \tau)\right\|_{L^{2}}^{2} \leq\left\|\rho^{\alpha}(\xi, \tau)\right\|_{L^{\infty}}^{2} \leq C\left\|\rho^{\alpha}(\xi, \tau)\right\|_{L^{2}}\left\|\left(\rho^{\alpha}\right)_{\xi}(\xi, \tau)\right\|_{L^{2}}
$$

which together with Young's inequality gives

$$
\left\|\rho^{\alpha}(\xi, \tau)\right\|_{L^{2}}^{2} \leq C\left\|\left(\rho^{\alpha}\right)_{\xi}(\xi, \tau)\right\|_{L^{2}}^{2},
$$

where $C$ is a positive constant independent of time. As $0<\alpha \leq \frac{1}{2}$ and as $\frac{1}{2}<\alpha \leq \gamma$, by means of the condition

$$
E_{0}^{\frac{1}{2}}\left(E_{0}+E_{1}+\left(\rho_{0}^{\gamma}\left(b_{0}\right)-\bar{\rho}^{\gamma}\right) b_{0}-\left(\rho_{0}^{\gamma}\left(a_{0}\right)-\bar{\rho}^{\gamma}\right) a_{0}\right)^{\frac{1}{2}} \leq \frac{v}{2(2 \alpha-1)} \bar{\rho}^{\frac{\gamma+2 \alpha-1}{2}},
$$

we can obtain two positive constants $\rho_{*}$ and $\rho^{*}$ independent of time such that

$$
0<\rho_{*} \leq \rho(\xi, \tau) \leq \rho^{*}, \quad(\xi, \tau) \in[0,1] \times[0, T], T>0
$$

The proof of this lemma is completed. 
We also have the regularity estimates for the solution $(\rho, u)$ to FBVP (3.2) as follows.

Lemma 3.4 Let $T>0$. Under the assumptions of Theorem 2.1, it holds for any strong solution $(\rho, u)$ to FBVP (3.2) that

$$
\left\{\begin{array}{l}
\rho \in L^{\infty}\left(0, T ; H^{1}([0,1])\right) \cap C^{0}([0,1] \times[0, T]), \\
u \in L^{\infty}\left(0, T ; H^{1}([0,1])\right) \cap L^{2}\left(0, T ; H^{2}([0,1])\right), \\
a(\tau), b(\tau) \in H^{1}([0, T]), \quad\left(\rho^{\gamma}-\rho^{1+\alpha} u_{\xi}\right) \in L^{\infty}\left(0, T ; L^{2}([0,1])\right) .
\end{array}\right.
$$

If it is also satisfied that

$$
u_{0} \in H^{2}([0,1])
$$

then the strong solution $(\rho, u)$ has the regularities

$$
\left\{\begin{array}{l}
(\rho, u) \in C^{0}([0,1] \times[0, T]), \\
\rho \in L^{\infty}\left(0, T ; H^{1}([0,1])\right), \quad \rho_{\tau} \in L^{\infty}\left(0, T ; L^{2}([0,1])\right), \\
u \in L^{\infty}\left(0, T ; H^{2}([0,1])\right) \cap L^{2}\left(0, T ; H^{3}([0,1])\right), \\
u_{\tau} \in L^{\infty}\left(0, T ; L^{2}([0,1])\right) \cap L^{2}\left(0, T ; H^{1}([0,1])\right), \\
a(\tau), b(\tau) \in H^{2}([0, T]), \quad\left(\rho^{\gamma}-\rho^{1+\alpha} u_{\xi}\right) \in C^{0}([0, T] \times([0,1])) .
\end{array}\right.
$$

Proof Multiplying $(3.2)_{2}$ by $\rho^{-(1+\alpha)} u_{\tau}$, integrating the result over $[0,1]$ and making use of the boundary conditions, after a direct computation and recombination, we have

$$
\begin{aligned}
\frac{d}{d \tau} \int_{0}^{1}\left(\frac{1}{2} u_{\xi}^{2}-\left(\rho^{\gamma}-\bar{\rho}^{\gamma}\right) \rho^{-(1+\alpha)} u_{\xi}\right) d \xi+\int_{0}^{1} \rho^{-(1+\alpha)} u_{\tau}^{2} d \xi \\
=[\gamma-(1+\alpha)] \int_{0}^{1} \rho^{\gamma-\alpha} u_{\xi}^{2} d \xi+(1+\alpha) \bar{\rho}^{\gamma} \int_{0}^{1} \rho^{-\alpha} u_{\xi}^{2} d \xi \\
\quad-(1+\alpha) \int_{0}^{1}\left(\rho^{\gamma}-\bar{\rho}^{\gamma}\right) \rho^{-(2+\alpha)} \rho_{\xi} u_{\tau} d \xi+(1+\alpha) \int_{0}^{1} \rho^{-1} \rho_{\xi} u_{\xi} u_{\tau} d \xi
\end{aligned}
$$

Integrating equation (3.44) over [0, $\tau]$, from (3.5), (3.7), (3.8) and (3.26), it is easily verified that

$$
\begin{aligned}
& \int_{0}^{1} u_{\xi}^{2} d \xi+\int_{0}^{\tau} \int_{0}^{1} u_{s}^{2} d \xi d s \\
& \quad \leq C+C \int_{0}^{\tau} \int_{0}^{1} u_{\xi}^{2} d \xi d s+C \int_{0}^{\tau} \int_{0}^{1} \rho_{\xi}^{2} d \xi d s+C \int_{0}^{\tau} \int_{0}^{1} \rho_{\xi}^{2} u_{\xi}^{2} d \xi d s
\end{aligned}
$$

where $C$ denotes a constant independent of time. From $(3.2)_{2},(3.5),(3.7),(3.8)$ and (3.26), we deduce

$$
\begin{aligned}
C \int_{0}^{\tau} \int_{0}^{1} \rho_{\xi}^{2} u_{\xi}^{2} d \xi d s \leq & \frac{C}{3} \int_{0}^{\tau} \int_{0}^{1} \rho_{\xi}^{2} u_{\xi}^{2} d \xi d s+\frac{1}{6} \int_{0}^{\tau} \int_{0}^{1} u_{s}^{2} d \xi d s \\
& +C \int_{0}^{\tau} \int_{0}^{1} \rho_{\xi}^{2} d \xi d s+C \int_{0}^{\tau} \int_{0}^{1} u_{\xi}^{2} d \xi d s .
\end{aligned}
$$


Using (3.46), we can obtain that

$$
\int_{0}^{1} u_{\xi}^{2} d \xi+\int_{0}^{\tau} \int_{0}^{1} u_{s}^{2} d \xi d s \leq C
$$

which together with $(3.2)_{2}$ implies

$$
\int_{0}^{1} u_{\xi}^{2} d \xi+\int_{0}^{\tau} \int_{0}^{1} u_{s}^{2} d \xi+\int_{0}^{\tau} \int_{0}^{1} u_{\xi \xi}^{2} d \xi d \tau \leq C
$$

Differentiating $(3.2)_{2}$ with respect to $\tau$, we get

$$
u_{\tau \tau}+\left(\rho^{\gamma}\right)_{\xi \tau}=\left(\rho^{1+\alpha} u_{\xi}\right)_{\xi \tau} .
$$

Taking product between (3.49) and $u_{\tau}$, integrating the results over $[0,1]$ and using the boundary conditions $(3.2)_{4}$, we have

$$
\frac{1}{2} \frac{d}{d \tau} \int_{0}^{1} u_{\tau}^{2} d \xi=\int_{0}^{1}\left(\rho^{\gamma}-\bar{\rho}^{\gamma}\right)_{\tau} u_{\xi \tau} d \xi-\int_{0}^{1}\left(\rho^{1+\alpha} u_{\xi}\right)_{\tau} u_{\xi \tau} d \xi
$$

The terms on the right-hand side of (3.50) can be bounded respectively as follows:

$$
\begin{aligned}
\int_{0}^{1}\left(\rho^{\gamma}-\bar{\rho}^{\gamma}\right)_{\tau} u_{\xi \tau} d \xi & =-\int_{0}^{1} \gamma \rho^{\gamma+1} u_{\xi} u_{\xi \tau} d \xi \\
& \leq-\frac{\gamma}{2} \frac{d}{d \tau} \int_{0}^{1} \rho^{\gamma+1} u_{\xi}^{2} d \xi+C \int_{0}^{1}\left(\rho^{1+\alpha} u_{\xi}^{2}+\rho^{2 \gamma-\alpha+3} u_{\xi}^{4}\right) d \xi
\end{aligned}
$$

and

$$
\begin{aligned}
-\int_{0}^{1}\left(\rho^{1+\alpha} u_{\xi}\right)_{\tau} u_{\xi \tau} d \xi & =-\int_{0}^{1}\left((1+\alpha) \rho^{\alpha} \rho_{\tau} u_{\xi}+\rho^{1+\alpha} u_{\xi \tau}\right) u_{\xi \tau} d \xi \\
& \leq-\frac{1}{2} \int_{0}^{1} \rho^{1+\alpha} u_{\xi \tau}^{2} d \xi+C \int_{0}^{1} \rho^{3+\alpha} u_{\xi}^{4} d \xi
\end{aligned}
$$

Summing (3.50)-(3.52) together and making use of (3.26) and (3.48), we obtain

$$
\begin{aligned}
& \frac{1}{2} \frac{d}{d \tau} \int_{0}^{1} u_{\tau}^{2} d \xi+\frac{\gamma}{2} \frac{d}{d \tau} \int_{0}^{1} \rho^{\gamma+1} u_{\xi}^{2} d \xi+\frac{1}{2} \int_{0}^{1} \rho^{1+\alpha} u_{\xi \tau}^{2} d \xi \\
& \leq C \int_{0}^{1} u_{\xi}^{2} d \xi+C\left\|u_{\xi}\right\|_{L^{\infty}([0,1])}^{2} \int_{0}^{1} u_{\xi}^{2} d \xi \\
& \leq C+C\left\|u_{\xi}\right\|_{L^{\infty}([0,1])}^{2} \int_{0}^{1} u_{\xi}^{2} d \xi .
\end{aligned}
$$

Integrating equation (3.53) over $[0, \tau]$, we get from $(3.2)_{2},(3.5)$ and $(3.26)$ that

$$
\begin{aligned}
& \int_{0}^{1} u_{\tau}^{2} d \xi+\int_{0}^{1} u_{\xi}^{2} d \xi+\int_{0}^{\tau} \int_{0}^{1} u_{\xi s}^{2} d \xi d s \\
& \leq C+C \sup _{\tau \in[0, T]}\left\|u_{\xi}\right\|_{L^{\infty}}^{2}
\end{aligned}
$$


Liana and Li Boundary Value Problems 2014, 2014:93

Page 12 of 15

http://www.boundaryvalueproblems.com/content/2014/1/93

$$
\begin{aligned}
& \leq C+C \sup _{\tau \in[0, T]}\left(\int_{0}^{1} u_{\xi}^{2} d \xi\right)^{\frac{1}{2}}\left(\int_{0}^{1} u_{\xi \xi}^{2} d \xi\right)^{\frac{1}{2}} \\
& \leq C+\frac{1}{2} \int_{0}^{1} u_{\tau}^{2} d \xi
\end{aligned}
$$

which gives

$$
\int_{0}^{1} u_{\tau}^{2} d \xi+\int_{0}^{1} u_{\xi}^{2} d \xi+\int_{0}^{T} \int_{0}^{1} u_{\xi \tau}^{2} d \xi d \tau \leq C
$$

which implies $\left(\rho^{\gamma}-\rho^{1+\alpha} u_{\xi}\right) \in L^{\infty}\left(0, T ; H^{1}([0,1])\right)$, and it follows from the definition of $a^{\prime}(\tau)=u(0, \tau)$ and $b^{\prime}(\tau)=u(1, \tau)$ that $a(\tau), b(\tau) \in H^{2}([0, T])$. The proof of this lemma is completed.

Finally, we give the large time behaviors of the strong solution as follows.

Lemma 3.5 Let $T>0$. Under the assumptions of Theorem 2.1, it holds for any strong soluion $(\rho, u)$ to FBVP (3.2) that

$$
\|(\rho-\bar{\rho}, u)(\cdot, \tau)\|_{L^{\infty}([0,1])} \leq C_{1} e^{-C_{2} \tau}, \quad \tau>0,
$$

where $C_{1}$ and $C_{2}$ denote two positive constants independent of time.

Proof Applying (3.6) and (3.12) with modification, we can obtain

$$
\frac{d}{d \tau} \int_{0}^{1}\left(\frac{1}{2} u^{2}+\frac{1}{\gamma-1}\left(\rho^{\gamma-1}-\bar{\rho}^{\gamma-1}\right)+\bar{\rho}^{\gamma}\left(\rho^{-1}-\bar{\rho}^{-1}\right)\right) d \xi+\int_{0}^{1} u_{\xi}^{2} d \xi \leq 0
$$

and

$$
\begin{aligned}
& \frac{d}{d \tau} \int_{0}^{1}\left(\frac{1}{2}\left(u+\frac{1}{\alpha}\left(\rho^{\alpha}\right)_{\xi}\right)^{2}+\frac{1}{\gamma-1}\left(\rho^{\gamma-1}-\bar{\rho}^{\gamma-1}\right)+\bar{\rho}^{\gamma}\left(\rho^{-1}-\bar{\rho}^{-1}\right)\right) d \xi+\int_{0}^{1} \rho_{\xi}^{2} d \xi \\
& \quad \leq-\left(\left(\rho^{\gamma}(1, \tau)-\bar{\rho}^{\gamma}\right) u(1, \tau)-\left(\rho^{\gamma}(0, \tau)-\bar{\rho}^{\gamma}\right) u(0, \tau)\right) .
\end{aligned}
$$

We have from (3.5), (3.7), (3.26), the Gagliardo-Nirenberg-Sobolev inequality and Young's inequality that

$$
\begin{aligned}
& \int_{0}^{1}\left(\frac{1}{\gamma-1}\left(\rho^{\gamma-1}-\bar{\rho}^{\gamma-1}\right)+\bar{\rho}^{\gamma}\left(\rho^{-1}-\bar{\rho}^{-1}\right)\right) d \xi \\
& \leq C \int_{0}^{1}(\rho-\bar{\rho})^{2} d \xi \leq C \int_{0}^{1} \rho_{\xi}^{2} d \xi \\
& \int_{0}^{1} u^{2} d \xi \leq C \int_{0}^{1} u_{\xi}^{2} d \xi
\end{aligned}
$$

and define

$$
E(\tau):=\int_{0}^{1}\left(\frac{1}{4} u^{2}+\frac{1}{4}\left(u+r\left(\rho^{\alpha}\right)_{\xi}\right)^{2}+\frac{1}{\gamma-1}\left(\rho^{\gamma-1}-\bar{\rho}^{\gamma-1}\right)+\bar{\rho}^{\gamma}\left(\rho^{-1}-\bar{\rho}^{-1}\right)\right) d \xi
$$


As the initial data satisfies (3.3), we have

$$
\left|\left(\rho^{\gamma}(1, \tau)-\bar{\rho}^{\gamma}\right) u(1, \tau)\right|+\left|\left(\rho^{\gamma}(0, \tau)-\bar{\rho}^{\gamma}\right) u(0, \tau)\right| \leq C e^{-\bar{C} \tau},
$$

where $C$ and $\bar{C}$ are positive constants independent of time. By (3.57)-(3.62), a complicated computation gives rise to

$$
\frac{d}{d \tau} E(\tau)+C_{0} E(\tau) \leq C e^{-\bar{C} \tau},
$$

where $C_{0}<\bar{C}$ is a positive constant independent of time. From (3.63), we have

$$
E(\tau) \leq E(0) e^{-C_{0} \tau}+C e^{-C_{0} \tau} \int_{0}^{\tau} e^{-\left(\bar{C}-C_{0}\right) s} d s \leq C e^{-C_{0} \tau} .
$$

As the initial data satisfies (3.4), from (3.22)-(3.24), we obtain

$$
\rho(1, \tau) \geq \rho(0, \tau) \geq \bar{\rho}>0,
$$

which implies that the domain $(b(\tau)-a(\tau))$ expands as $t$ grows up, so that we have

$$
b^{\prime}(\tau)-a^{\prime}(\tau)=u(1, \tau)-u(0, \tau) \geq 0 .
$$

Then it holds from (3.65) and (3.66) that

$$
\left(\rho^{\gamma}(1, \tau)-\bar{\rho}^{\gamma}\right) u(1, \tau)-\left(\rho^{\gamma}(0, \tau)-\bar{\rho}^{\gamma}\right) u(0, \tau) \geq 0 .
$$

Using (3.58), after a complicated computation, we have

$$
\frac{d}{d \tau} E(\tau)+C_{0} E(\tau) \leq 0
$$

where $C_{0}$ is a positive constant independent of time, and we have

$$
E(\tau) \leq E(0) e^{-C_{0} \tau} \leq C e^{-C_{0} \tau},
$$

where $C$ is a positive constant independent of time.

By the fact

$$
E(\tau) \geq c\left(\|(\rho-\bar{\rho}, u)\|_{L^{2}([0,1])}^{2}+\left\|\left(\rho^{\alpha}\right)_{\xi}\right\|_{L^{2}([0,1])}^{2}\right)
$$

where $c>0$ is a constant independent of time, and the Gagliardo-Nirenberg-Sobolev inequality

$$
\|(\rho-\bar{\rho}, u)\|_{L^{\infty}([0,1])} \leq\|(\rho-\bar{\rho}, u)\|_{L^{2}([0,1])}^{\frac{1}{2}}\left\|(\rho-\bar{\rho}, u)_{\xi}\right\|_{L^{2}([0,1])}^{\frac{1}{2}},
$$

we can deduce (3.56). 


\section{Proof of the main results}

Proof The global existence of a unique strong solution to FBVP (1.1) and (2.1) can be established in terms of the short time existence carried out as in [7], the uniform a priori estimates and the analysis of regularities, which indeed follow from Lemmas 3.1-3.4. We omit the details. The large time behaviors follow from Lemma 3.5 directly. The proof of Theorem 2.1 is completed.

\section{Competing interests}

The authors declare that they have no competing interests.

\section{Authors' contributions}

All authors contributed to each part of this work equally. All authors read and approved the final manuscript.

\section{Author details}

${ }^{1}$ College of Mathematics and Information Science, North China University of Water Resources and Electric Power Zhengzhou, 450011, P.R. China. ${ }^{2}$ Institute of Atmospheric Physics, Chinese Academy of Sciences, Beijing, 100029, P.R. China. ${ }^{3}$ College of Teacher Education, Quzhou University, Quzhou, 324000, P.R. China.

\section{Acknowledgements}

The authors thank the referee for the helpful comments and suggestions on the paper. The research of Ruxu Lian is supported by NNSFC No. 11101145, China Postdoctoral Science Foundation No. 2012M520360, Doctoral Foundation of North China University of Water Sources and Electric Power No. 201032, Innovation Scientists and Technicians Troop Construction Projects of Henan Province. The research of Jian Liu is supported by NNSFC No. 11326140, the Doctoral Starting up Foundation of QuZhou University No. BSYJ201314.

Received: 8 January 2014 Accepted: 2 April 2014 Published: 06 May 2014

\section{References}

1. Gerbeau, JF, Perthame, B: Derivation of viscous Saint-Venant system for laminar shallow water, numerical validation. Discrete Contin. Dyn. Syst., Ser. B 1, 89-102 (2001)

2. Marche, F: Derivation of a new two-dimensional viscous shallow water model with varying topography, bottom friction and capillary effects. Eur. J. Mech. B, Fluids 26, 49-63 (2007)

3. Bresch, D, Desjardins, B: Existence of global weak solutions for a $2 \mathrm{D}$ viscous shallow water equations and convergence to the quasi-geostrophic model. Commun. Math. Phys. 238, 211-223 (2003)

4. Bresch, D, Desjardins, B: On the construction of approximate solutions for the 2D viscous shallow water model and for compressible Navier-Stokes models. J. Math. Pures Appl. 86, 362-368 (2006)

5. Fang, D, Zhang, T: Global solutions of the Navier-Stokes equations for compressible flow with density-dependent viscosity and discontinuous initial data. J. Differ. Equ. 222, 63-94 (2006)

6. Guo, Z, Li, H, Xin, Z: Lagrange structure and dynamics for solutions to the spherically symmetric compressible Navier-Stokes equations. Commun. Math. Phys. 309, 371-412 (2012)

7. Jiang, S, Xin, Z, Zhang, P: Global weak solutions to 1D compressible isentropy Navier-Stokes with density-dependent viscosity. Methods Appl. Anal. 12, 239-252 (2005)

8. Lian, R, Guo, Z, Li, H: Dynamical behaviors of vacuum states for 1D compressible Navier-Stokes equations. J. Differ. Equ. 248, 1926-1954 (2010)

9. Liu, J: Local existence of solution to free boundary value problem for compressible Navier-Stokes equations. Acta Math. Sci., Ser. B 32, 1298-1320 (2012)

10. Liu, T, Xin, Z, Yang, T: Vacuum states for compressible flow. Discrete Contin. Dyn. Syst. 4, 1-32 (1998)

11. Okada, M, Nečasoá, ŠM, Makino, T: Free boundary problem for the equation of one-dimensional motion of compressible gas with density-dependent viscosity. Ann. Univ. Ferrara, Sez. 7: Sci. Mat. 48, 1-20 (2002)

12. Vong, S, Yang, T, Zhu, C: Compressible Navier-Stokes equations with degenerate viscosity coefficient and vacuum (II). J. Differ. Equ. 192, 475-501 (2003)

13. Yang, T, Yao, Z, Zhu, C: Compressible Navier-Stokes equations with density-dependent viscosity and vacuum. Commun. Partial Differ. Equ. 26, 965-981 (2001)

14. Yang, T, Zhao, H: A vacuum problem for the one-dimensional compressible Navier-Stokes equations with density-dependent viscosity. J. Differ. Equ. 184, 163-184 (2002)

15. Yang, T, Zhu, C: Compressible Navier-Stokes equations with degenerate viscosity coefficient and vacuum. Commun. Math. Phys. 230, 329-363 (2002)

16. Mellet, A, Vasseur, A: Existence and uniqueness of global strong solutions for one-dimensional compressible Navier-Stokes equations. SIAM J. Math. Anal. 39, 1344-1365 (2008)

17. Guo, Z, Jiu, Q, Xin, Z: Spherically symmetric isentropic compressible flows with density-dependent viscosity coefficients. SIAM J. Math. Anal. 39, 1402-1427 (2008)

18. Jiu, Q, Wang, Y, Xin, Z: Stability of rarefaction waves to the $1 \mathrm{D}$ compressible Navier-Stokes equations with density-dependent viscosity. Commun. Partial Differ. Equ. 36, 602-634 (2011)

19. Jiu, Q, Xin, Z: The Cauchy problem for 1D compressible flows with density-dependent viscosity coefficients. Kinet. Relat. Models 1, 313-330 (2008)

20. Li, H, Li, J, Xin, Z: Vanishing of vacuum states and blow-up phenomena of the compressible Navier-Stokes equations. Commun. Math. Phys. 281, 401-444 (2008) 
21. Lian, R, Liu, J, Li, H, Xiao, L: Cauchy problem for the one-dimensional compressible Navier-Stokes equations. Acta Math. Sci., Ser. B 32, 315-324 (2012)

22. Xin, Z: Blowup of smooth solutions to the compressible Navier-Stokes equation with compact density. Commun. Pure Appl. Math. 51, 229-240 (1998)

23. Solonnikov, VA, Tani, A: Free boundary problem for a viscous compressible flow with a surface tension. In: Constantin Carathéodory: An International Tribute, vol. II, pp. 1270-1303. World Scientific, Teaneck (1991)

24. Zajaczkowski, WM: Existence of local solutions for free boundary problems for viscous compressible barotropic fluids. Ann. Pol. Math. 60, 255-287 (1995)

25. Zajaczkowski, WM: On nonstationary motion of a compressible barotropic viscous fluid bounded by a free surface. Diss. Math. 324, 1-101 (1993)

26. Secchi, P, Valli, A: A free boundary problem for compressible viscous fluids. J. Reine Angew. Math. 341, 1-31 (1983)

27. Tani, A: On the free boundary value problem for compressible viscous fluid motion. J. Math. Kyoto Univ. 21, 839-859 (1981)

28. Zadrzyńska, E, Zajaczkowski, WM: On local motion of a general compressible viscous heat conducting fluid bounded by a free surface. Ann. Pol. Math. 59, 133-170 (1994)

29. Solonnikov, VA, Tani, A: Evolution free boundary problem for equations of motion of viscous compressible barotropic liquid. In: The Navier-Stokes Equations II - Theory and Numerical Methods (Oberwolfach, 1991). Lecture Notes in Math., vol. 1530, pp. 30-55. Springer, Berlin (1992)

30. Zajaczkowski, WM: On nonstationary motion of a compressible barotropic viscous capillary fluid bounded by a free surface. SIAM J. Math. Anal. 25, 1-84 (1994)

31. Zadrzyńska, E: Evolution free boundary problem for equations of viscous compressible heat-conducting capillary fluids. Math. Methods Appl. Sci. 24, 713-743 (2001)

32. Zadrzyńska, E, Zajaczkowski, WM: On the global existence theorem for a free boundary problem for equations of a viscous compressible heat conducting capillary fluid. J. Appl. Anal. 2, 125-169 (1996)

10.1186/1687-2770-2014-93

Cite this article as: Lian and Liu: On the free boundary value problem for one-dimensional compressible

Navier-Stokes equations with constant exterior pressure. Boundary Value Problems 2014, 2014:93

\section{Submit your manuscript to a SpringerOpen ${ }^{\odot}$ journal and benefit from:}

- Convenient online submission

- Rigorous peer review

- Immediate publication on acceptance

- Open access: articles freely available online

- High visibility within the field

- Retaining the copyright to your article 